\title{
Erratum to: Risk-averse dynamic programming for Markov decision processes
}

\section{Andrzej Ruszczyński}

Published online: 10 May 2014

(C) Springer-Verlag Berlin Heidelberg and Mathematical Optimization Society 2014

\section{Erratum to: Math. Program., Ser. B (2010) 125:235-261 DOI 10.1007/s10107-010-0393-3}

The Original publication of the article suffers from a technical inaccuracy associated with verification of measurability of the optimal decision rules. This erratum corrects the gap, by introducing additional conditions of joint continuity and measurability of the functions and multifunctions involved.

We use the terminology and notation of [4].

A controlled kernel $Q$ is setwise (strongly) continuous, if for all bounded measurable functions $v$ on $\mathscr{X}$, and all convergent sequences $\left\{\left(x_{k}, u_{k}\right)\right\}, k=1,2, \ldots$, one has

$$
\lim _{k \rightarrow \infty} \int_{\mathscr{X}} v(y) Q\left(d y \mid x_{k}, u_{k}\right)=\int_{\mathscr{X}} v(y) Q(d y \mid x, u),
$$

where $x=\lim _{k \rightarrow \infty} x_{k}$ and $u=\lim _{k \rightarrow \infty} u_{k}$. In the product space $\mathscr{X} \times \mathscr{M}$, we always consider the product topology of strong convergence in $\mathscr{X}$ and the above setwise convergence of measures in $\mathscr{M}$. Observe that in the case when $Q(\cdot \mid x, u) \in$ $\mathscr{M} \subset \mathscr{Y}=\mathscr{L}_{q}\left(\mathscr{X}, \mathscr{B}, P_{0}\right)$ for all $(x, u)$, the setwise continuity and the weak* continuity [with $v \in \mathscr{V}=\mathscr{L}_{p}\left(\mathscr{X}, \mathscr{B}, P_{0}\right)$ in (1)] are equivalent.

We make the following general assumptions (for $t=1,2, \ldots$ ):

The online version of the original article can be found under doi:10.1007/s10107-010-0393-3.

A. Ruszczyński $(\bowtie)$

Department of Management Science and Information Systems,

Rutgers University, Piscataway, NJ 08854, USA

e-mail: rusz@business.rutgers.edu 
G1. The transition kernels $Q_{t}(\cdot, \cdot)$ are strongly continuous;

G2. The conditional risk measures $\rho_{t}$ are Markov and the multifunctions $\mathscr{A}_{t}(\cdot, \cdot) \equiv$ $\partial_{\varphi} \sigma_{t}(0, \cdot, \cdot)$ are lower semicontinuous;

G3. The functions $c_{t}(\cdot, \cdot)$ are bounded and lower semicontinuous;

G4. The multifunctions $U_{t}(\cdot)$ are measurable and compact-valued.

First, we establish semicontinuity of a risk transition mapping with respect to the state-control pair.

Proposition 1 Suppose (G1)-(G3) and let $v \in \mathscr{V}$ be bounded. Then the mapping $(x, u) \mapsto \sigma_{t}\left(v, x, Q_{t}(x, u)\right)$ is lower semicontinuous on $\operatorname{graph}\left(U_{t}\right)$.

Proof Consider the dual representation of the risk transition mapping

$$
\sigma_{t}\left(v, x, Q_{t}(x, u)\right)=\max _{\mu \in \mathscr{A}_{t}\left(x, Q_{t}(x, u)\right)} \int_{\mathscr{X}} v(y) \mu(d y) .
$$

By (G1) and (G2), the multifunction $(x, u) \mapsto \mathscr{A}_{t}\left(x, Q_{t}(x, u)\right)$ is lower semicontinuous. Owing to condition (G3), the function $(x, u, \mu) \mapsto \int_{\mathscr{X}} v(y) \mu(d y)$ is lower semicontinuous on graph $\left(U_{t}\right) \times \mathscr{M}$. The assertion follows now from [1, Theorem 1.4.16], whose proof remains valid in our setting as well.

If the risk transition mapping $\sigma(\cdot, \cdot, \cdot)$ is continuous, then its subdifferential $\partial_{\varphi} \sigma_{t}(0, \cdot, \cdot)$ is upper semicontinuous. However, in Proposition 1 , we assume lower semicontinuity of the mapping $(x, m) \mapsto \partial_{\varphi} \sigma(0, x, m)$, which is not trivial and should be verified for each case.

Example 1 Let us verify the lower semicontinuity assumption for the multifunction $\mathscr{A}$ given in [4, Example 4] with $r=1$ :

$$
\begin{gathered}
\mathscr{A}(x, m)=\left\{\mu \in \mathscr{M}: \exists\left(h \in \mathscr{L}_{\infty}\left(\mathscr{X}, \mathscr{B}, P_{0}\right)\right) \frac{d \mu}{d m}=1+h-\langle h, m\rangle,\right. \\
\left.\|h\|_{\infty} \leq \kappa, h \geq 0\right\} .
\end{gathered}
$$

Consider an arbitrary $\mu \in \mathscr{A}(x, m)$ and suppose $x_{k} \rightarrow x, m_{k} \rightarrow m$, as $k \rightarrow \infty$. We need to find $\mu_{k} \in \mathscr{A}\left(x_{k}, m_{k}\right)$ such that $\mu_{k} \rightarrow \mu$. Let $h$ be the function for which, according to (3), $\frac{d \mu}{d m}=1+h-\int h(z) m(d z)$. We define $\mu_{k}$ by specifying their Radon-Nikodym derivatives: $\frac{d \mu_{k}}{d m_{k}}=1+h-\int h(z) m_{k}(d z)$. By construction, $\mu_{k} \in \mathscr{A}\left(x_{k}, m_{k}\right)$. Then, for any bounded measurable function $v$, we obtain

$$
\begin{aligned}
\int_{\mathscr{X}} v(y) \mu_{k}(d y) & =\int_{\mathscr{X}} v(y)\left(1+h(y)-\int_{\mathscr{X}} h(z) m_{k}(d z)\right) m_{k}(d y) \\
& =\int_{\mathscr{X}} v(y)(1+h(y)) m_{k}(d y)-\int_{\mathscr{X}} h(z) m_{k}(d z) \int_{\mathscr{X}} v(y) m_{k}(d y) .
\end{aligned}
$$


As $m_{k} \rightarrow m$, we conclude that

$$
\begin{aligned}
\lim _{k \rightarrow \infty} \int_{\mathscr{X}} v(y) \mu_{k}(d y) & =\int_{\mathscr{X}} v(y)(1+h(y)) m(d y)-\int_{\mathscr{X}} h(z) m(d z) \int_{\mathscr{X}} v(y) m(d y) \\
& =\int_{\mathscr{X}} v(y) \mu(d y),
\end{aligned}
$$

which is the strong convergence of $\mu_{k}$ to $\mu$.

Recall that a normal integrand is a function $f: \mathscr{X} \times U \rightarrow \mathbb{R} \cup\{+\infty\}$ such that that its epigraphical mapping $x \mapsto\{(u, \alpha) \in \mathscr{U} \times \mathbb{R}: f(x, u) \leq \alpha\}$ is a closed-valued and measurable multifunction [3, sec. 14.D].

Proposition 2 Suppose (G1)-(G4) and let $v: \mathscr{X} \rightarrow \mathbb{R}$ be measurable and bounded. Then for all $t=1,2 \ldots$ the functions

$$
\psi_{t}(x)=\inf _{u \in U_{t}(x)}\left\{c_{t}(x, u)+\sigma_{t}\left(v, x, Q_{t}(x, u)\right)\right\}, \quad x \in \mathscr{X},
$$

are measurable and bounded, and measurable selectors $\pi_{t}$ of $U_{t}$ exist, such that

$$
\psi_{t}(x)=c_{t}\left(x, \pi_{t}(x)\right)+\sigma_{t}\left(v, x, Q_{t}\left(x, \pi_{t}(x)\right)\right), \quad \forall x \in \mathscr{X} .
$$

Proof Let us fix $t$ and consider the function $f_{t}: \mathscr{X} \times U \rightarrow \mathbb{R} \cup\{+\infty\}$ defined as follows:

$$
f_{t}(x, u)= \begin{cases}c_{t}(x, u)+\sigma_{t}\left(v, x, Q_{t}(x, u)\right) & \text { if } u \in U_{t}(x) \\ +\infty & \text { otherwise }\end{cases}
$$

Owing to Proposition $1, f_{t}(\cdot, \cdot)$ is lower semicontinuous and is thus a normal integrand [3, Ex. 14.31]. It follows from [3, Thm. 14.37] that the function $\psi_{t}(x)=\inf _{u} f_{t}(x, u)$ is measurable and that the optimal solution mapping $\Psi_{t}(x)=\left\{u \in U_{t}(x): \psi_{t}(x)=\right.$ $\left.f_{t}(x, u)\right\}$ is measurable. By $(\mathrm{G} 4)$, the set $U_{t}(x)$ is compact, and thus $\Psi_{t}(x) \neq \varnothing$ for all $x \in \mathscr{X} . \Psi_{t}$ is also compact-valued. By virtue of [2], a measurable selector $\pi_{t}$ of $\Psi_{t}$ exists. Let us recall the dual representation (2) again:

$$
\psi_{t}(x)=c_{t}\left(x, \pi_{t}(x)\right)+\max _{\mu \in \mathscr{A}_{t}\left(x, Q_{t}\left(x, \pi_{t}(x)\right)\right)} \int_{\mathscr{X}} v(y) \mu(d y) .
$$

As the set $\mathscr{A}_{t}\left(x, Q_{t}\left(x, \pi_{t}(x)\right)\right)$ contains only probability measures, and the functions $c_{t}(\cdot, \cdot)$ and $v(\cdot)$ are bounded, the function $\psi_{t}(\cdot)$ is bounded as well.

In [4, Thm. 2], the assumptions (i)-(v) have to be replaced by the following conditions:

(i) The general conditions (G1)-(G4) are satisfied;

(ii) The function $c_{T+1}(\cdot)$ is measurable and bounded. 
The assertion is the same as before, with the additional claim that the value functions $v_{t}(\cdot)$ are measurable and bounded.

The proof of [4, Thm. 2] follows the original line of argument. The only difference is that at the bottom of page 247 , where [3, Thm. 14.37] is invoked, we need to invoke Proposition 2 from this note. The conditions (i)-(ii) above have to be assumed also in [4, Cor. 2], which uses Theorem 2.

In [4, Thm. 4], the assumptions (i)-(iv) have to be replaced by the following conditions:

(i') The general conditions (G1)-(G4) are satisfied;

(ii') The conditional risk measures $\rho_{t}, t=1,2, \ldots$, are stationary.

The assertion is the same as before, with the additional claim that the value function $\hat{v}(\cdot)$ is measurable and bounded.

The proof of [4, Thm. 4] remains unchanged; the conditions (G1)-(G4) are needed because Theorem 2 is invoked. Originally, [4, Thm. 4] assumed also nonnegativity of the cost function $c(\cdot, \cdot)$, but this condition not needed, if (G3) is assumed, as explained on page 251 of [4]. The conditions (i')-(ii') above have to be assumed also in [4, Thms. 5-7 and Cor. 3], all of which use Theorem 4.

Acknowledgments The author thanks Eugene Feinberg for pointing out a gap in [4, Thm. 2].

\section{References}

1. Aubin, J.-P., Frankowska, H.: Set-Valued Analysis. Birkhäuser, Boston (1990)

2. Kuratowski, K., Ryll-Nardzewski, C.: A general theorem on selectors. Bulletin de l'Académie Polonaise de Sciences, Série Mathématiques, Astronomie et Physique 13, 397-411 (1965)

3. Rockafellar, R.T., Wets, R.J.-B.: Variational Analysis. Springer, Berlin (1998)

4. Ruszczyński, A.: Risk-averse dynamic programming for Markov decision processes. Math. Progr. Ser. B 125, 235-261 (2010) 